PROCEEDINGS OF THE

AMERICAN MATHEMATICAL SOCIETY

Volume 135, Number 4, April 2007, Pages 997-999

S 0002-9939(06)08570-4

Article electronically published on October 13, 2006

\title{
SIGN CHANGES OF HECKE EIGENVALUES OF SIEGEL CUSP FORMS OF GENUS TWO
}

\author{
WINFRIED KOHNEN
}

(Communicated by Ken Ono)

\begin{abstract}
We shall show that the eigenvalues of a Hecke eigenform of integral weight and genus 2 not contained in the Maass space change signs infinitely often.
\end{abstract}

\section{Introduction AND STATEMENT OF THE RESUlt}

Let $f$ be a nonzero cusp form of integral weight $k$ and level $N$ with real Fourier coefficients $a(n)(n \in \mathbf{N})$. Using a classical theorem of Landau [7] together with the analytic properties of the Hecke $L$-function and Rankin-Selberg zeta function of $f$ (in particular the fact that the Hecke $L$-series of $f$ has finite abscissa of convergence), it is not hard to show that the sequence $(a(n))_{n \in \mathbf{N}}$ has infinitely many sign changes; i.e., there are infinitely many $n$ with $a(n)>0$ as well as $a(n)<0$ (for an extension to more general subgroups of $S L_{2}(\mathbf{R})$ and a discussion of related questions, cf. 6] ). In particular, this applies when $f$ is a normalized Hecke eigenform and so $a(n)$ is equal to the $n$-th Hecke eigenvalue of $f$.

The above result easily generalizes to the Fourier coefficients of a nonzero cusp form $F$ of integral weight $k$ on the Siegel modular group $\Gamma_{g}:=S p_{g}(\mathbf{Z}) \subset G L_{2 g}(\mathbf{Z})$ of genus $g>1$ when these coefficients are real. For the proof one uses the analytic properties of the Koecher-Maass Dirichlet series and the Rankin-Selberg Dirichlet series attached to $F$ (cf. e.g. 2, 8).

For $g>1$, however, it seems that from this one cannot deduce any assertions about sign changes of Hecke eigenvalues of $F$ if $F$ is a Hecke eigenform, since for $g>1$ the eigenvalues are no longer "proportional" (in any reasonable sense) to the Fourier coefficients. Recall that the eigenvalues again are real, since the Hecke operators are Hermitian with respect to the Petersson scalar product.

Indeed, if $g=2$ and $k$ is even and we denote by $\lambda_{n}$ the eigenvalue of $F$ under the usual Hecke operator $T_{n}(n \in \mathbf{N})$ [1, then it is well known that the Hecke eigenform $F$ is contained in the Maass subspace $S_{k}^{*}\left(\Gamma_{2}\right)$ if and only if $\lambda_{n}>0$ for all $n$. 3 . For the Maass subspace $S_{k}^{*}\left(\Gamma_{2}\right)$, cf. e.g. [4]. In particular recall that the $T_{n}$ leave $S_{k}^{*}\left(\Gamma_{2}\right)$ invariant.

The purpose of this paper is to show the following.

Received by the editors July 26, 2005 and, in revised form, November 15, 2005. 2000 Mathematics Subject Classification. Primary 11F46.

(C)2006 American Mathematical Society 
Theorem 1. Let $F$ be a nonzero Hecke eigenform of integral weight $k$ on $\Gamma_{2}$ with Hecke eigenvalues $\lambda_{n}(n \in \mathbf{N})$. Suppose that $F$ lies in the orthogonal complement of $S_{k}^{*}\left(\Gamma_{2}\right)$ if $k$ is even. Then the sequence $\left(\lambda_{n}\right)_{n \in \mathbf{N}}$ has infinitely many sign changes.

We note that as is well known, the first odd $k$ where $S_{k}\left(\Gamma_{2}\right)$ is nonzero is $k=35$, and the first even $k$ for which the orthogonal complement of $S_{k}^{*}\left(\Gamma_{2}\right)$ is nonzero, is $k=20$ (in both cases, the corresponding spaces are one-dimensional).

The proof of the theorem, which will be given in the next section, is based again on Landau's theorem coupled with the analytic properties of the spinor zeta function of $F$ [ and a theorem of Weissauer 10 that asserts the truth of the generalized Ramanujan-Petersson conjecture for $F$ as in the theorem.

\section{ProOF}

Recall that

$$
\sum_{n \geq 1} \lambda_{n} n^{-s}=\zeta(2 s-2 k+4)^{-1} Z_{F}(s) \quad(\Re(s)>>0)
$$

where

$$
Z_{F}(s)=\prod_{p} Z_{F, p}\left(p^{-s}\right)^{-1} \quad(\Re(s)>>0)
$$

is the spinor zeta function of $F$. Here

$$
Z_{F, p}(X)=\left(1-\alpha_{0, p} X\right)\left(1-\alpha_{1, p} X\right)\left(1-\alpha_{2, p} X\right)\left(1-\alpha_{0, p} \alpha_{1, p} \alpha_{2, p} X\right)
$$

and $\alpha_{0, p}, \alpha_{1, p}$ and $\alpha_{2, p}$ are "the" Satake $p$-parameters of $F$. The completed function

$$
Z_{F}^{*}(s)=(2 \pi)^{-2 s} \Gamma(s) \Gamma(s-k+2) Z_{F}(s)
$$

has meromorphic continuation to $\mathbf{C}$ and is $(-1)^{k}$-invariant under $s \mapsto 2 k-2-s$ [1. Moreover, $Z_{F}^{*}(s)$ is entire if either $k$ is odd or if $k$ is even and $F$ is contained in the orthogonal complement of $S_{k}^{*}\left(\Gamma_{2}\right)[5,[9]$.

To prove the theorem, it is sufficient to assume that $\lambda_{n} \geq 0$ for all but finitely many $n$ and to derive a contradiction.

Under this assumption, we deduce from Landau's theorem [7 that the Dirichlet series on the left of (1) either converges for all $s \in \mathbf{C}$ or has a singularity at the real pont of its line of convergence.

As is well known, $\zeta(s)$ has no zeros on the real line except at the points $s=$ $-2 m(m \in \mathbf{N})$, and the latter zeros are simple.

Since $Z_{F}^{*}(s)$ is entire, $Z_{F}(s)$ is entire and in particular vanishes at the points $s=k-2, k-3, \ldots$ Hence the function on the right-hand side of (1) has no singularity on the real line. We therefore conclude that

$$
\zeta(2 s-2 k+4)^{-1} Z_{F}(s)
$$

must be entire.

Now choose $s_{0} \in \mathbf{C}$ such that $\Re\left(2 s_{0}-2 k+4\right)=\frac{1}{2}$ and $\zeta\left(2 s_{0}-2 k+4\right)=0$. Then $Z_{F}(s)$ must vanish at $s=s_{0}$.

On the other hand, under our assumption on $F$, by Weissauer's theorem 10 the Satake $p$-parameters $\alpha_{1, p}, \alpha_{2, p}$ are of absolute value 1 . Since

$$
\alpha_{0, p}^{2} \alpha_{1, p} \alpha_{2, p}=p^{2 k-3}
$$


[1, we conclude that the Euler product for $Z_{F}(s)$ converges absolutely for $\Re(s)>$ $k-\frac{1}{2}$ and so $Z_{F}(s) \neq 0$ in this range. Applying the functional equation

$$
Z_{F}^{*}(2 k-2-s)=(-1)^{k} Z_{F}^{*}(s)
$$

and evaluating at $s=s_{0}$, we obtain a contradition since $\Re\left(2 k-2-s_{0}\right)=k-\frac{1}{4}$.

\section{REFERENCES}

[1] A. N. Andrianov: Euler products corresponding to Siegel modular forms of genus 2, Russ. Math. Surv. 29, 45-116 (1974) MR0432552 (55:5540)

[2] S. Böcherer and S. Raghavan: On Fourier coefficients of Siegel modular forms, J. Reine Angew. Math. 384, 80-101 (1988). MR0929979 (89c:11068)

[3] S. Breulmann: On Hecke eigenforms in the Maass space, Math. Z. 232, no. 3, 527-530 (1999). MR:1719682 (2000j:11066)

[4] M. Eichler and D. Zagier: The theory of Jacobi forms, Progress in Math, vol. 55, Birkhäuser: Boston, 1985. MR0781735 (86j:11043)

[5] S. A. Evdokimov: A characterization of the Maass space of Siegel cusp forms of genus 2 (in Russian), Mat. Sbornik (154) 112, 133-142 (1980). MR0575936 (82a:10028)

[6] M. Knopp, W. Kohnen and W. Pribitkin: On the signs of Fourier coefficients of cusp forms, The Ramanujan J. 7, 269-277 (2003). MR2035806 (2004m:11064)

[7] E. Landau, Über einen Satz von Tschebyschef, Math. Ann. 61, 527-550 (1906).

[8] H. Maass, Siegel's modular forms and Dirichlet series, Lect. Not. in Math. 216, Springer: Berlin, 1971. MR0344198 (49:8938)

[9] T. Oda: On modular forms associated with indefinite quadratic forms of signature $(2, n-2)$, Math. Ann. 231, 97-144 (1977). MR0466026 (57:5909)

[10] R. Weissauer: The Ramanujan conjecture for genus 2 Siegel modular forms (an application of the trace formula). Preprint, Mannheim (1993).

Mathematisches Institut, Universität Heidelberg, INF 288, D-69120 Heidelberg, GerMANY

E-mail address: winfried@mathi.uni-heidelberg.de 